\title{
Linking Thin Flux Tube MHD Models to Apparent Stellar Surface Activity
}

\author{
T. Granzer \\ Astrophysikalisches Institut Potsdam, An der Sternwarte 16, D-14482 \\ Potsdam, Germany \\ K. G. Strassmeier \\ Astrophysikalisches Institut Potsdam, An der Sternwarte 16, D-14482 \\ Potsdam, Germany
}

\begin{abstract}
We model thin magnetic flux tubes as they rise from the bottom of a stellar convection zone to the photosphere. On emergence they form active regions, i.e. star spots. This model was very successfully applied to the solar case, where the simulations where in agreement with the butterfly diagram, Joy's law, and Hale's law. We propose the use of a similar model to describe stellar activity in the more extreme form found on active stars. A comparison between Doppler-images of wellobserved pre-MS stars and a theoretically derived probability of star-spot formation as a function of latitude is presented.
\end{abstract}

\section{Introduction}

We used the well-known thin flux-tube approximation (e.g. Spruit 1981) to model the ascent of magnetic flux from the overshoot region to the surface. A flux tube in an initial stable mechanic equilibrium inside the overshoot region is exposed to a small (undulatory) disturbance which eventually grows and leaps out of the overshoot region into the convective envelope. Here, the superadiabatic stratification amplifies the growth of the disturbance which evolves into a (single) rising loop. The simulation must cease when the thin-flux-tube approximation breaks down due to the vast drop of the pressure scale height near the surface layer. In all cases studied, the flux-tube summits reach, however, an elevation of approximately $0.98 \mathrm{R}_{\star}$. The early end of the simulations prohibits any model-based predictions on post-emergence development of the star spots. One can merely argue that meridional flows may transport magnetic flux to the poles, than backing this assumptions with actual simulations. Velocity fields below the surface layers can be taken into account, but for all stars other than our Sun our knowledge is very limited. Thus, all simulations are carried out with a rigid rotational profile. For the Sun, it was shown in Caligari, Moreno-Insertis, \& Schüssler (1995)that adopting a differential rotation law resembling our Sun's rotational profile does not alter the emergence path of the flux tubes.

For each model star, twelve individual flux tubes with different equilibrium latitudes inside the overshoot layer $\left(5^{\circ}\right.$ to $\left.60^{\circ}\right)$ are considered. The end-points 


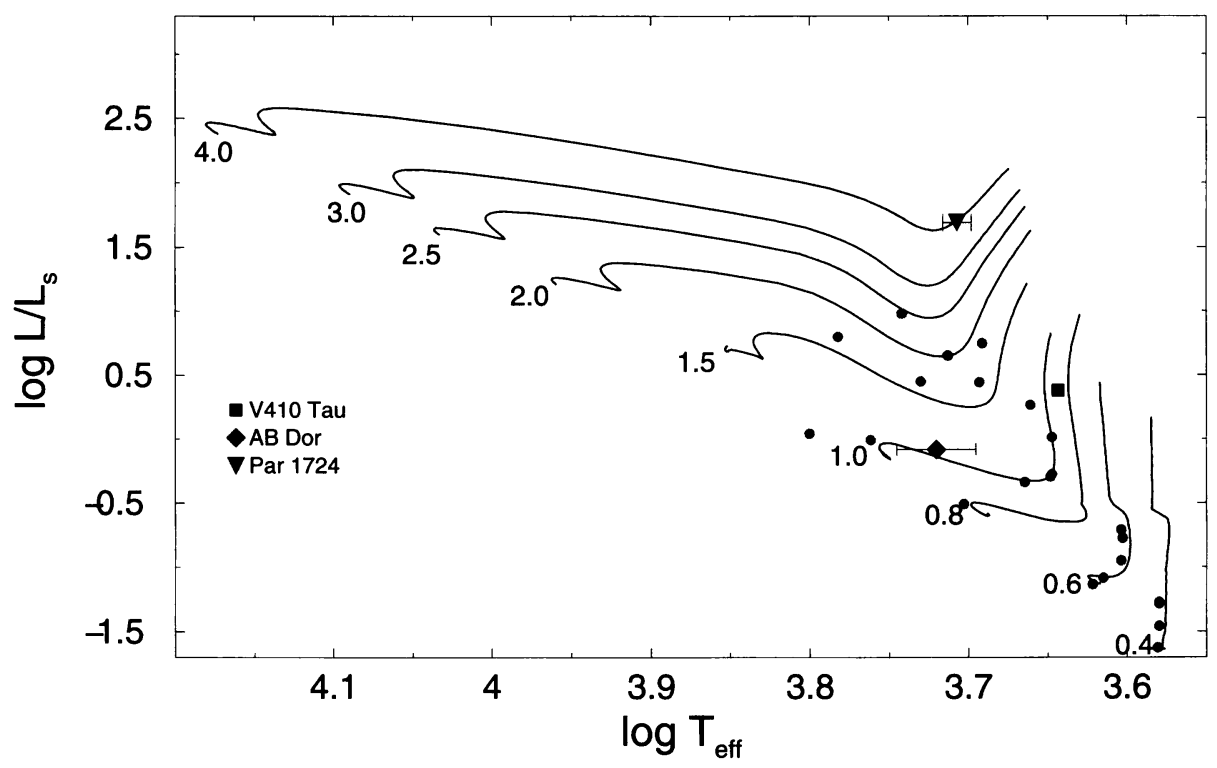

Figure 1. An HR-diagram showing the position of the stars presented. The tracks given are theoretically derived pre-MS evolution of stars with masses between 0.4 and $4 \mathrm{M}_{\odot}$. Models used in the coresize comparison, Fig. 2, are given as dots. Stars with an direct comparison between the theoretical spot distribution and a surface image are labeled accordingly. The error bars given their, if any, are the errors in the temperature and luminosity determination as given by the respective authors.

of the numerical simulation of all twelve flux tubes are combined to yield the spot probability function, SPF, a normalized measure of probability of starspot formation as a function of latitude. Latitude zones with a wide SPF correspond to latitudes with high probability of spot formation (see, e.g. Fig. 2).

The importance of the Coriolis force on the emergence path of magnetic flux tubes rising through an outer convection zone was realized very early, see e.g. Schüssler \& Solanki (1992), Choudhuri \& Gilman (1987), or Fan, Fischer, \& DeLuca (1993). For the Sun, a satisfying numerical model was proposed by Caligari et al. (1995). In an attempt to extend this model to solar-like stars, Schüssler et al. (1996) and Granzer et al. (2000) showed again the importance of the Coriolis force by establishing a clear rule between high stellar rotation rate and high-latitude flux emergence. Another important footprint of the Coriolis force is the influence of the relative size of the radiative core. For a handful of stars (refer also to Fig. 1) we show this influence in Fig. 2. All model stars rotate at a fixed rotation rate of $\Omega=10 \Omega_{\odot}$. For extremely small cores, i.e. for $R_{c} / R_{*} \lesssim 0.3$, the flux tubes detaches from the overshoot layer. In this case, the Coriolis force only balances the magnetic tension and does not influence the emergence path. Consequentially, we have flux tube emergence at all latitudes. In stars with intermediate to large core sizes, i.e. for $R_{c} / R_{*} \gtrsim 0.3$, a considerable 


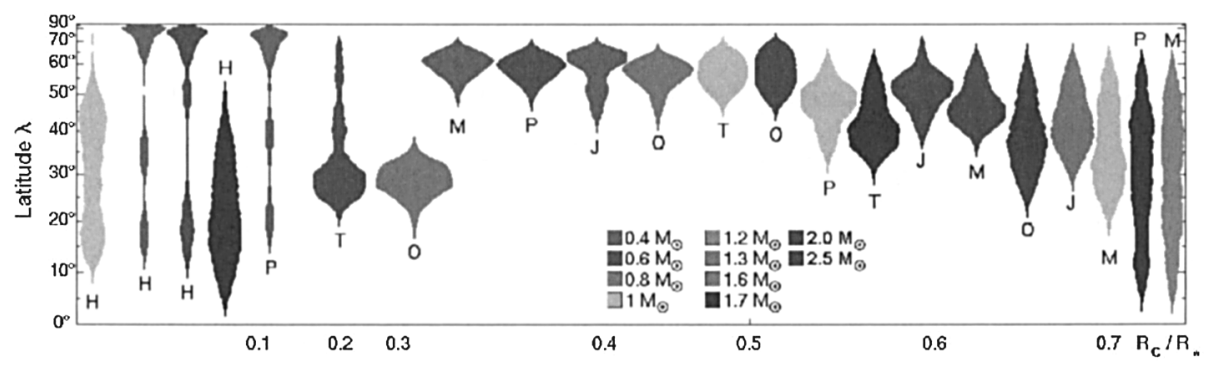

Figure 2. The dependency of spot latitude on relative core size for a fixed rotation rate of $\Omega=10 \Omega_{\odot}$. Latitude zones with a wide spotprobability function (SPF, see text) correspond to zones with an enhanced probability of spot formation. The transition at $R_{c} / R_{*} \approx 0.3$ marks the transition between detached flux tubes found in stars with small cores to anchored flux tubes found in stars with intermediate to large cores.

part of the flux tube remains anchored in the overshoot layer and the Coriolis force acting on the rising part of the flux tube now counteracts the buoyancy force in the plane perpendicular to the axis of rotation, resulting in a strong poleward force. Consequentially, the surfacing latitude of the flux tube drops with increasing core size.

\section{Comparison to Doppler-Images}

An important test for any theory is a validation with observations. For our Sun, the model was able to explain various properties of sun spots, see Caligari et al. (1995) for details. In the stellar case, the best test imaginable right now is a comparison of the proposed spot latitudes with observed surface maps.

Table 1. Parameters of the stars with a given comparison between the theoretically derived spot probability and a surface map reconstructed with Doppler imaging techniques. The mass, age, luminosity, temperature, and rotation rate given follow the values stated by the respective authors.

\begin{tabular}{lllllll}
\hline Star & Spec. & $M / \mathrm{M}_{\odot}$ & age $/ \mathrm{Myr}$ & $L / \mathrm{L}_{\odot}$ & $T_{\text {eff }} / \mathrm{K}$ & $\Omega / \Omega_{\odot}$ \\
\hline V410 Tau & K5 & 1.0 & 1.0 & 2.4 & 4400 & 14.1 \\
AB Dor & K1IIIp & 0.9 & 15 & 0.9 & 5250 & 51.2 \\
Par 1724 & G8n & 3.0 & 0.5 & 49 & 4850 & 5.68 \\
\hline \hline
\end{tabular}

For our comparison of we picked three well-observed, young stars. Their stellar properties can be found in Tab. 1, their position in a theoretical HRdiagram along with relevant PMS-tracks is given in Fig. 1. For all of the stars presented, a stellar model matching their predicted mass and age has been used. 

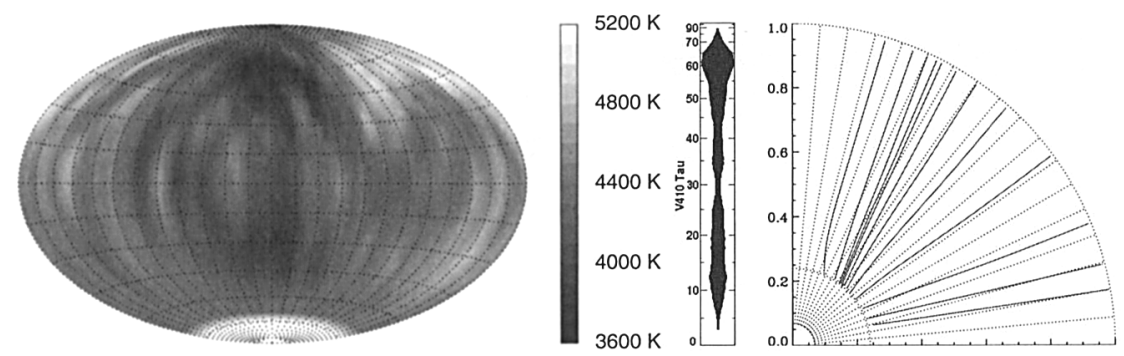

Figure 3. Comparison of a Doppler image of V410 Tau (Rice \& Strassmeier 1996), left panel, with the theoretical spot probability, central panel, and the surfacing path of the flux tubes, right panel. Both, the high-latitude feature centered slightly above $60^{\circ}$ and the lowlatitude features close to the equator are reproduced in the theoretical spot probability.

The rotation rate applied is the photometric rotation rate. Note that the theoretical model does not distinguish between northern and southern hemispheres, therefore only one hemisphere is shown.

\subsection{V410 Tau}

V410 is a WTTS with a proposed mass of one solar mass and an age of one Myr. The rotational period is $\mathrm{P}=1.872095 \mathrm{~d}$, yielding a rotation rate of roughly 14 times solar. The comparison of the Doppler image taken from Rice \& Strassmeier (1996), Fig. 3, shows an excellent agreement of the theoretical map with the observed surface. The spot probability peaks at $60^{\circ}$, exactly coinciding with the biggest spot in the map. The low latitude features visible have their counterpart in the second maximum of the SPF at $10^{\circ}$.

\subsection{AB Dor}

AB Dor has been extensively studied and the spot latitudes reported are rather divers. While Kürster, Schmitt, \& Cutispoto (1994) report low-latitude spots, Unruh, Colier-Cameron, \& Cutispoto (1995) a year later find low-latitude spots together with a polar feature. Another four years later, Donati et al. (1999) find only features above $60^{\circ}$. In Fig. 4 we give the comparison of the theory with the map given in Unruh et al. (1995). Although the similarities between the surface map and the SPF is not striking, we want to point out the very different rise path of the flux tubes, Fig. 4, rightmost panel. Flux tubes at low latitudes rise almost vertical, due to their weak magnetic field, while flux tubes at higher latitudes rise almost radially. Although their emergence latitude is almost identically, this two distinct groups may account for the two activity zones on AB Dor. Flux tubes emerging from close to the equator may be responsible for the polar spot. Due to their weaker magnetic field they have a less pronounced magnetic tension and are thus not as rigid as the flux tubes from high latitudes. This higher flexibility makes them also more susceptible to post-emergence motions, allowing in principle for a fast transport to the pole. 

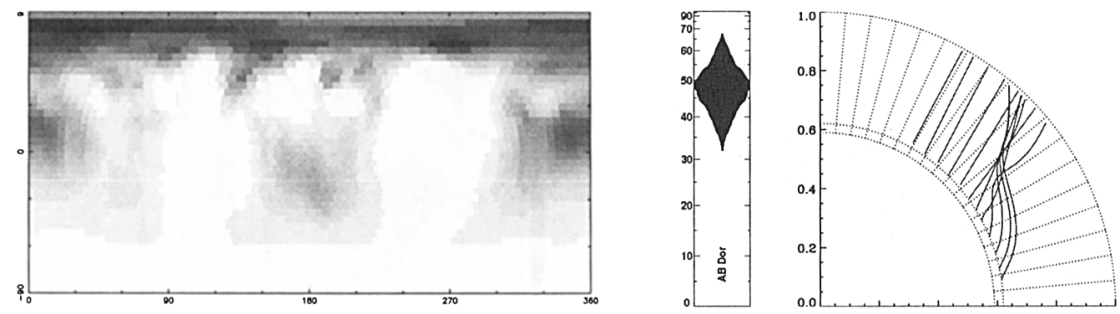

Figure 4. Comparison of a Doppler image of AB Dor (Unruh, ColierCameron, \& Cutispoto 1995), left panel, with the theoretical spot probability, central panel, and the surfacing path of the flux tubes, right panel. The Doppler map shows features at higher latitude than the theoretically derived spot probability. The low-latitude features, too, do not have an analogon in the SPF.

\subsection{Par $\mathbf{1 7 2 4}$}

Par 1724 is a star with a relatively high mass, $3 \mathrm{M}_{\odot}$. It has an outer convection zone only because of its small age, 500000 yrs. The Doppler image shown in Fig. 5 is taken from Neuhäuser et al. (1998). The theory predicts concentrated flux emergence around $35^{\circ}$ which coincides well with the central latitude of the biggest spot, but does not account for the large extension of the spot.
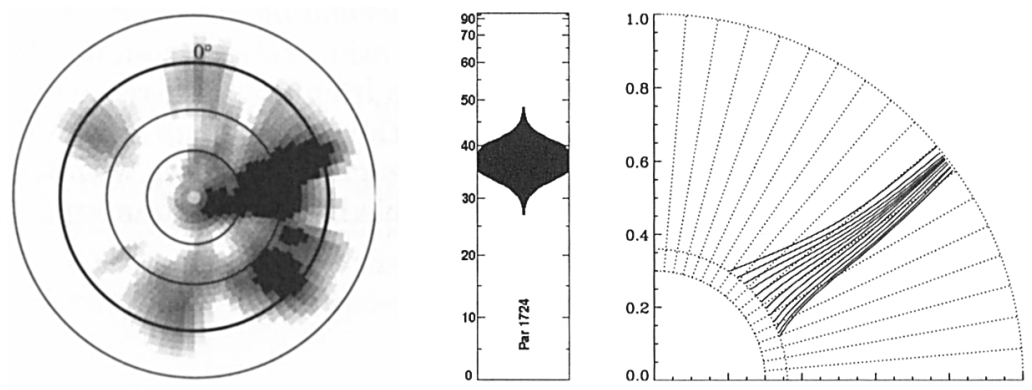

Figure 5. Comparison of a Doppler image of Par 1724 (Neuhäuser et al. 1998), left panel, with the theoretical spot probability, central panel, and the surfacing path of the flux tubes, right panel. The extended feature centered at approximately $30^{\circ}$ is very well reproduced by the theoretical model.

\section{Discussion and Conclusions}

If one wants to directly compare our simulation results to surface maps derived with Doppler imaging, care must be taken at certain points. First, our model is not capable of predicting post-emergence evolution of the star spots. It might well be that surface meridional flow, though unimportant on our Sun, may divert the spots once they have formed. As long as the life-time of the spot is big compared to the drift time, the apparent surface image may be in utterly 
disagreement with the spot probability derived from the numerical model (see, e.g. subsection 2.2 on AB Dor). Furthermore, the spot probability we derive does not distinguish between different stages during possible activity cycles on other stars. Even for our Sun, a single snap-shot of its spot distribution may not be fitted too well with our model, though considering an averaged surface map covering an entire activity cycle does. For most of the stars present here, only a few or even only a single Doppler maps are available, meaning that we are in the ill-posed situation of having only a snap-shot available and no clear understanding of spot locations averaged over an entire stellar activity cycle.

Considering all these caveats, the results of the numerical model are in good agreement to the currently available observations. This suggests, in turn, that the dynamo acting in these very active stars is not qualitatively different to the proposed main dynamo of our Sun - an interface dynamo acting at the bottom of the convection zone. Observational techniques that reveal the geometry of the surface magnetic field also address this question of similarities in the dynamo of our Sun and other stars. First results, e.g. on LQ Hya and HR 1099 (Donati, 1999) indicate that the surface magnetic field there has a strong toroidal component, in contrast to the surface magnetic field of our Sun. Nevertheless, the resolution of these maps is not high enough to rule out the possibility that the strong radial field one expects from surfacing flux tubes cancel out in the reconstructed image due to the different polarity found in a single active region. Further observations with higher spatial resolution are required to come to a distinctive conclusion here.

\section{References}

Caligari, P., Moreno-Insertis, F., \& Schüssler, M. 1995, ApJ, 441, 886

Choudhuri, A. R., \& Gilman, P. A. 1987, ApJ, 316, 788

Donati, J.-F. 1999, MNRAS, 302, 457

Donati, J.-F., Collier-Cameron, A., Hussain, G. A. J., \& Semel, M. 1999, MNRAS, 302, 437

Fan, Y., Fisher, G., \& DeLuca, E. E. 1993, ApJ, 405, 390

Granzer, Th., Caligari, P., Schüssler, M., \& Strassmeier, K. G. 2000, A\&A, 355, 1087

Kürster, M., Schmitt, J. H. M. M., \& Cutispoto, G. 1994, A\&A289, 899

Neuhäuser, R., Wolk, S. J., Torres, G., et al. 1998, A\&A, 334, 873

Rice, J. B., \& Strassmeier, K. G. 1996, A\&A, 316, 164

Schüssler, M., Caligari, P., Ferriz-Mas, A., Solanki, S. K., \& Stix, M. 1996, A\&A, 314, 503

Schüssler, M., \& Solanki, S. K. 1992, A\&A, 264, L13

Spruit, H. C. 1981, A\&A, 98, 155

Unruh, Y. C., Collier-Cameron, A., \& Cutispoto, G. 1995, MNRAS, 277, 1145 